\title{
Expansive actions on uniform spaces and surjunctive maps
}

\author{
Tullio Ceccherini-Silberstein • Michel Coornaert
}

Received: 11 January 2011 / Revised: 4 February 2011 / Accepted: 9 February 2011 /

Published online: 9 March 2011

(C) The Author(s) 2011. This article is published with open access at SpringerLink.com

\begin{abstract}
We present a uniform version of a result of M. Gromov on the surjunctivity of maps commuting with expansive group actions and discuss several applications. We prove in particular that for any group $\Gamma$ and any field $\mathbb{K}$, the space of $\Gamma$-marked groups $G$ such that the group algebra $\mathbb{K}[G]$ is stably finite is compact.
\end{abstract}

Keywords Expansive action - Uniform space - Surjunctive map ·

Cellular automaton · Shift action · Subshift · Group algebra $\cdot$ Stable finiteness

Mathematics Subject Classification (2000) $\quad 54 \mathrm{E} 15 \cdot 37 \mathrm{~B} 15 \cdot 68 \mathrm{Q} 80$

\section{Introduction}

A map $f$ from a set $X$ into itself is said to be surjunctive if it is surjective or not injective [14]. Thus, a non-surjunctive map is a map which is injective but not surjective. With this terminology at hand, Dedekind's characterization of infinite sets may be rephrased by saying that a set $X$ is infinite if and only if it admits a non-surjunctive map $f: X \rightarrow X$. Similarly, elementary linear algebra tells us that a vector space is infinite-dimensional if and only if it admits a non-surjunctive endomorphism. In fact, it

Communicated by E. Zelmanov.

T. Ceccherini-Silberstein $(\varangle)$

Dipartimento di Ingegneria, Università del Sannio, C.so Garibaldi 107, 82100 Benevento, Italy e-mail: tceccher@mat.uniroma1.it

M. Coornaert

Institut de Recherche Mathématique Avancée, UMR 7501, Université de Strasbourg et CNRS,

7 rue René-Descartes, 67000 Strasbourg, France

e-mail: coornaert@math.unistra.fr 
turns out that the absence of non-surjunctive endomorphisms for a given mathematical object $X$ often reflects some "finiteness" property of $X$.

The word surjunctive was created by Gottschalk [13] who introduced the notion of a surjunctive group. Let $G$ be a group. Given a set $A$, consider the set $A^{G}$ equipped with the prodiscrete topology, that is, with the product topology obtained by taking the discrete topology on each factor $A$ of $A^{G}$. There is a natural action of $G$ on $A^{G}$ obtained by shifting coordinates via left multiplication in $G$ (see Sect. 5). This action is called the $G$-shift and the study of its dynamical properties is the central theme of symbolic dynamics. The group $G$ is said to be surjunctive if, for any finite set $A$, every $G$-equivariant continuous map $\tau: A^{G} \rightarrow A^{G}$ is surjunctive. Gottschalk asked whether every group $G$ is surjunctive. Although there is a large class of groups, namely sofic groups (see [11,19]), which are known to be surjunctive, this question remains open. Here, it is worth mentioning that the existence of a non-sofic group is also an open problem.

In algebraic geometry, there is a famous theorem of Ax [1] which says that every endomorphism of a complex algebraic variety is surjunctive. Ax' surjunctivity theorem was extended by Gromov [14] to certain classes of "symbolic algebraic spaces" obtained by taking projective limits of algebraic varieties. In order to establish these generalizations, Gromov developed various techniques for proving surjunctivity of maps $f: X \rightarrow X$ obtained as limits of surjunctive maps $f_{i}: X_{i} \rightarrow X_{i}$. He also used one of these techniques to prove the closedness of the set of marked surjunctive groups (see $[12,14]$ for an alternative proof based on model theory). The purpose of our paper is to give a detailed exposition of this technique in a somewhat more general setting that the one considered in [14]. To be a little bit more precise, we extend results on the surjunctivity of maps commuting with expansive actions on metric spaces by replacing metric spaces by uniform spaces. It turns out that this extension is quite natural and we think it may help to a better understanding of the roles of the various mathematical concepts involved in Gromov's approach.

Uniform spaces were introduced by Weil [18] as a generalization of metric spaces and topological groups. In a uniform space $X$, the closeness of a pair of points is not measured by a real number, like in a metric space, but by the fact that this pair of points belong or does not belong to certain subsets of the Cartesian square $X \times X$ which are called the entourages of the uniform structure (see Sect. 2 for precise definitions). Every metric on a set $X$ defines a uniform structure on $X$ and every uniform structure defines a topology on the underlying set. In the case when the uniform structure comes from a metric, the associated topology coincides with the topology defined by the metric.

Uniformly continuous maps between uniform spaces are defined as being the maps which pullback entourages to entourages.

There is a natural uniform structure on the set $\mathcal{P}(X)$ of subsets of a uniform space $X$, which is called the Hausdorff-Bourbaki uniform structure. Its name comes from the fact that it generalizes the Hausdorff distance on the set of bounded closed subsets of a metric space and was first mentioned in exercises in the treatise of Bourbaki [2].

The classical definition of expansivity for groups actions on metric spaces may be extended to uniform spaces. More precisely, an action of a group $\Gamma$ on a uniform space 
$X$ is said to be expansive if there is an entourage $V_{0}$ such that there is no pair of distinct points whose orbit under the diagonal action of $\Gamma$ on $X \times X$ remains inside $V_{0}$.

One of our main results is the following theorem whose proof will be given in Sect. 4:

Theorem 1.1 Let $X$ be a uniform space equipped with a uniformly continuous and expansive action of a group $\Gamma$ and let $f: X \rightarrow X$ be a uniformly continuous and $\Gamma$-equivariant map. Let $Y$ be a compact subset of $X$. Suppose that there exists a net $\left(Z_{i}\right)_{i \in I}$ of $\Gamma$-invariant subsets of $X$, which converges to $Y$ in the Hausdorff-Bourbaki topology such that $f\left(Z_{i}\right) \subset Z_{i}$ and the restriction maps $\left.f\right|_{Z_{i}}: Z_{i} \rightarrow Z_{i}$ are surjunctive for all $i \in I$. Then $Y$ is $\Gamma$-invariant, $f(Y) \subset Y$, and the restriction map $\left.f\right|_{Y}: Y \rightarrow Y$ is surjunctive.

As an immediate consequence of the preceding theorem, we get

Corollary 1.2 Let $X$ be a uniform space equipped with a uniformly continuous and expansive action of a group $\Gamma$ and let $f: X \rightarrow X$ be a uniformly continuous and $\Gamma$-equivariant map. Let $\Sigma(f)$ denote the set of $\Gamma$-invariant compact subsets $Y \subset X$ such that $f(Y) \subset Y$ and the restriction map $\left.f\right|_{Y}: Y \rightarrow Y$ is surjunctive. Then $\Sigma(f)$ is closed in the set of all subsets of $X$ for the Hausdorff-Bourbaki topology.

Consider now a group $G$ and an arbitrary set $A$. There is a natural uniform structure on $A^{G}$ which is called the prodiscrete uniform structure (see Sect. 2.3). The associated topology is the prodiscrete topology on $A^{G}$ and the shift action is expansive and uniformly continuous. Note that if $G$ is uncountable then the prodiscrete topology on $A^{G}$ is not metrizable as soon as $A$ has at least two elements. The uniformly continuous $G$-equivariant maps $\tau: A^{G} \rightarrow A^{G}$ are precisely the cellular automata over the group $G$ and the alphabet $A$ (see Sect. 5).

Let $\Gamma$ be a group. The set of quotients of $\Gamma$ may be identified with the set $\mathcal{N}(\Gamma)$ of normal subgroups of $\Gamma$. The set $\mathcal{N}(\Gamma)$ is called the set of $\Gamma$-marked groups. It is a closed (and hence compact) subset of the compact space $\mathcal{P}(\Gamma)=\{0,1\}^{\Gamma}$ for the prodiscrete topology.

By applying Theorem 1.1 to the shift on $A^{\Gamma}$ with $A$ finite, we deduce in Sect. 6 the above mentioned closedness theorem for surjunctive groups:

Corollary 1.3 (Gromov) Let $\Gamma$ be a group. Then the set of normal subgroups $N \subset \Gamma$ such that the quotient group $\Gamma / N$ is surjunctive is closed (and hence compact) in $\mathcal{N}(\Gamma)$.

Let now $\mathbb{K}$ be a field. A group $G$ is said to be $\mathrm{L}_{\mathbb{K}}$-surjunctive if, for any finitedimensional vector space $V$ over $\mathbb{K}$, every linear cellular automaton $\tau: V^{G} \rightarrow V^{G}$ is surjunctive. It is known that every sofic group is $\mathrm{L}_{\mathbb{K}}$-surjunctive (see [4]). In Sect. 7 , we shall establish the closedness of the set of $\mathrm{L}_{\mathbb{K}}$-surjunctive marked groups:

Theorem 1.4 Let $\Gamma$ be a group and let $\mathbb{K}$ be a field. Then the set of normal subgroups $N \subset \Gamma$ such that the quotient group $\Gamma / N$ is $L_{\mathbb{K}}$-surjunctive is closed (and hence compact) in $\mathcal{N}(\Gamma)$. 
The proof of the preceding theorem uses a stronger version of Theorem 1.1 (see Corollary 4.2), where the compactness hypothesis for $Y$ is replaced by the weaker hypotheses that both $Y$ and $f(Y)$ are closed in $X$ together with the fact that the restriction of $f$ to $Y$ is a uniform embedding.

One of the famous conjectures of Kaplansky [16] about the algebraic properties of group algebras is that $\mathbb{K}[G]$ is stably finite for any group $G$ and any field $\mathbb{K}$ (we recall that a ring $R$ is said to be stably finite if every one-sided invertible square matrix over $R$ is also two-sided invertible). This conjecture remains open up to now although it was settled by Kaplansky himself for all fields of characteristic 0 and later by Elek and Szabó [10] (see also [4]) for sofic groups in any characteristic. In [4], it is shown that a group $G$ is $\mathrm{L}_{\mathbb{K}}$-surjunctive if and only if the group algebra $\mathbb{K}[G]$ is stably finite. We thus have:

Corollary 1.5 Let $\Gamma$ be a group and let $\mathbb{K}$ be a field. Then the set of normal subgroups $N \subset \Gamma$ such that the group algebra $\mathbb{K}[\Gamma / N]$ is stably finite is closed (and hence compact) in $\mathcal{N}(\Gamma)$.

The paper is organized as follows. Section 2 contains background material on uniform spaces. The definition of the Hausdorff-Bourbaki uniform structure on the set of subsets of a uniform space is recalled in Sect. 3. For the convenience of the reader, proofs of some well known facts about the Hausdorff-Bourbaki uniform structure that are needed in the sequel are also included in this section. In Sect. 4 we prove a uniform version of Lemma 4.H in [14] which is used to prove Theorem 1.1. Basic properties of cellular automata are presented in Sect. 5. Section 6 is devoted to marked groups. We show that if $G=\Gamma / N$ is a $\Gamma$-marked group, then the set $A^{G}$ is canonically isomorphic, as a uniform $G$-space, to the subset $\operatorname{Fix}(N) \subset A^{\Gamma}$ consisting of the points of $A^{\Gamma}$ which are fixed by the normal subgroup $N \subset \Gamma$. We also prove that the map $N \mapsto \operatorname{Fix}(N)$ yields a uniform embedding of $\mathcal{N}(\Gamma)$, the space of $\Gamma$-marked groups, into $\mathcal{P}\left(A^{\Gamma}\right)$. This gives a symbolic representation of the space of $\Gamma$ marked groups which is used, in combination with Theorem 1.1 (resp. Corollary 1.2), in the proof of Corollary 1.3 (resp. Theorem 1.4) which is completed in Sect. 6 (resp. Sect. 7).

\section{Uniform structures}

In this section we collect some basic facts about uniform spaces, uniformly continuous maps, and uniformly continuous group actions. More details and proofs may be found for example in [2,15], or [17].

Throughout the paper, we shall use the following general notation. Let $X$ be a set. We denote by $\mathcal{P}(X)$ the set of all subsets of $X$ and by $\Delta_{X}$ the diagonal in $X \times X$, that is, $\Delta_{X}=\{(x, x): x \in X\}$. Let $R$ be a binary relation on $X$, that is, a subset $R \subset X \times X$. Given an element $y \in X$, we define the subset $R[y] \subset X$ by $R[y]=\{x \in X:(x, y) \in R\}$. For $Y \subset X$, we define the set $R[Y] \subset X$ by $R[Y]=\bigcup_{y \in Y} R[y]$, so that we have in particular $R[y]=R[\{y\}]$ for all $y \in X$. 
The inverse ${ }^{-1}$ of $R$ is the binary relation ${ }^{-1} R=\{(x, y):(y, x) \in R\}$. One says that

$R$ is symmetric if $\stackrel{-1}{R}=R$.

The composite of two binary relations $R$ and $S$ is the binary relation

$R \circ S=\{(x, y):$ there exists $z \in X$ such that $(x, z) \in R$ and $(z, y) \in S\} \subset X \times X$.

\subsection{Uniform spaces}

Definition Let $X$ be a set. A uniform structure on $X$ is a non-empty set $\mathcal{U}$ of subsets of $X \times X$ satisfying the following conditions:

(UN-1) if $V \in \mathcal{U}$, then $\Delta_{X} \subset V$;

(UN-2) if $V \in \mathcal{U}$ and $V \subset V^{\prime} \subset X \times X$, then $V^{\prime} \in \mathcal{U}$;

(UN-3) if $V \in \mathcal{U}$ and $W \in \mathcal{U}$, then $V \cap W \in \mathcal{U}$;

(UN-4) if $V \in \mathcal{U}$, then -1

(UN-5) if $V \in \mathcal{U}$, then there exists $W \in \mathcal{U}$ such that $W \circ W \subset V$.

A set $X$ equipped with a uniform structure $\mathcal{U}$ is called a uniform space and the elements of $\mathcal{U}$ are called the entourages of $X$.

Let $(X, \mathcal{U})$ be a uniform space. If $Y$ is a subset of $X$, then $\mathcal{U}_{Y}=\{V \cap(Y \times Y)$ : $V \in \mathcal{U}$ \} is a uniform structure on $Y$, which is said to be induced by $\mathcal{U}$.

A subset $\mathcal{B} \subset \mathcal{U}$ is called a base of $\mathcal{U}$ if for each $V \in \mathcal{U}$ there exists $B \in \mathcal{B}$ such that $B \subset V$.

It is easy to see that a base $\mathcal{B}$ satisfies the following properties:

(B-1) if $V \in \mathcal{B}$, then $\Delta_{X} \subset V$;

(B-2) if $V \in \mathcal{B}$ and $W \in \mathcal{B}$, then there exists $U \in \mathcal{B}$ such that $U \subset V \cap W$;

(B-3) if $V \in \mathcal{B}$, then there exists $W \in \mathcal{B}$ such that $W \subset-\frac{1}{V}$;

(B-4) if $V \in \mathcal{B}$, then there exists $W \in \mathcal{B}$ such that $W \circ W \subset V$.

Conversely, given a set $X$ and a subset $\mathcal{B} \subset \mathcal{P}(X \times X)$ satisfying conditions (B-1)(B-4), there exists a unique uniform structure $\mathcal{U}$ on $X$ admitting $\mathcal{B}$ as a base.

There is a topology on $X$ which is associated with the uniform structure. This is the topology for which the neighborhoods of an arbitrary point $x \in X$ consist of the sets $V[x]$, where $V$ runs over all entourages of $X$. This topology is Hausdorff if and only if the intersection of the entourages of $X$ coincides with the diagonal $\Delta_{X} \subset X \times X$.

Examples (1) The discrete uniform structure on a set $X$ is the uniform structure whose entourages consist of all subsets of $X \times X$ containing $\Delta_{X}$. The topology associated with this uniform structure is the discrete topology on $X$.

(2) If $(X, d)$ is a metric space, then there is a uniform structure on $X$ associated with the metric. A base for this uniform structure is given by the sets $V_{\varepsilon}=\{(x, y) \in$ $X \times X: d(x, y)<\varepsilon\}, \varepsilon>0$. The topology associated with this uniform structure coincides with the topology associated with the metric. 


\subsection{Uniformly continuous maps}

Let $X$ and $Y$ be uniform spaces.

A map $f: X \rightarrow Y$ is said to be uniformly continuous if it satisfies the following condition: for each entourage $W$ of $Y$, there exists an entourage $V$ of $X$ such that $(f \times f)(V) \subset W$. Here $f \times f$ denotes the map from $X \times X$ into $Y \times Y$ defined by $(f \times f)\left(x_{1}, x_{2}\right)=\left(f\left(x_{1}\right), f\left(x_{2}\right)\right)$ for all $\left(x_{1}, x_{2}\right) \in X \times X$. Every uniformly continuous map $f: X \rightarrow Y$ is continuous but the converse fails to hold in general.

A uniform isomorphism between uniform spaces $X$ and $Y$ is a bijective map $f: X \rightarrow Y$ such that both $f$ and $f^{-1}$ are uniformly continuous. One says that a map $f: X \rightarrow Y$ is a uniform embedding if $f$ is injective and induces a uniform isomorphism between $X$ and $f(X)$.

\subsection{Product of uniform spaces}

Suppose that $\left(X_{\lambda}\right)_{\lambda \in \Lambda}$ is a family of uniform spaces. Then the smallest uniform structure on the Cartesian product $X=\prod_{\lambda \in \Lambda} X_{\lambda}$ for which all projection maps $\pi_{\lambda}: X \rightarrow$ $X_{\lambda}, \lambda \in \Lambda$, are uniformly continuous is called the product uniform structure on $X$. A base of entourages for the product uniform structure on $X$ is obtained by taking all subsets of $X \times X$ which are of the form

$$
\begin{aligned}
\prod_{\lambda \in \Lambda} V_{\lambda} & \subset \prod_{\lambda \in \Lambda} X_{\lambda} \times X_{\lambda} \\
& =\left(\prod_{\lambda \in \Lambda} X_{\lambda}\right) \times\left(\prod_{\lambda \in \Lambda} X_{\lambda}\right) \\
& =X \times X,
\end{aligned}
$$

where $V_{\lambda} \subset X_{\lambda} \times X_{\lambda}$ is an entourage of $X_{\lambda}$ and $V_{\lambda}=X_{\lambda} \times X_{\lambda}$ for all but finitely many $\lambda \in \Lambda$.

If $Y$ is a uniform space, then a map $f: Y \rightarrow X$ is uniformly continuous if and only if the maps $\pi_{\lambda} \circ f: Y \rightarrow X_{\lambda}$ are uniformly continuous for all $\lambda \in \Lambda$.

In the particular case when each $X_{\lambda}$ is endowed with the discrete uniform structure, the product uniform structure on $X=\prod_{\lambda \in \Lambda} X_{\lambda}$ is called the prodiscrete uniform structure on $X$.

\subsection{Uniformly continuous and expansive actions}

Suppose that $X$ is a uniform space equipped with an action of a group $\Gamma$.

One says that the action of $\Gamma$ on $X$ is uniformly continuous if the map $f_{\gamma}: X \rightarrow X$ defined by $f_{\gamma}(x)=\gamma x$ is uniformly continuous for each $\gamma \in \Gamma$.

Consider the diagonal action of $\Gamma$ on $X \times X$ defined by $\gamma(x, y)=(\gamma x, \gamma y)$ for all $x, y \in X$ and $\gamma \in \Gamma$. One says that the action of $\Gamma$ on $X$ is expansive if there exists an entourage $W_{0}$ of $X$ satisfying the following property: for any two distinct points $x, y \in X$, there exists an element $\gamma \in \Gamma$ such that $\gamma(x, y) \notin W_{0}$. In other words, the 
action of $\Gamma$ on $X$ is expansive if and only if there exists an entourage $W_{0}$ such that

$$
\bigcap_{\gamma \in \Gamma} \gamma^{-1} W_{0}=\Delta_{X}
$$

Note that if $X$ admits a uniformly continuous and expansive action then the topology on $X$ is necessarily Hausdorff.

\section{The Hausdorff-Bourbaki uniform structure}

In this section we briefly review the definition and basic properties of the HausdorffBourbaki uniform structure on the set of subsets of a uniform space (see [2, ch. II exerc. 5 p. 34 and exerc. 6 p. 36]). Some proofs of well known facts that will be used in the next section are included for the convenience of the reader.

Let $X$ be a uniform space with uniform structure $\mathcal{U}$. For $V \in \mathcal{U}$, we define the subset $\widehat{V} \subset \mathcal{P}(X) \times \mathcal{P}(X)$ by

$$
\widehat{V}=\{(Y, Z) \in \mathcal{P}(X) \times \mathcal{P}(X): Z \subset V[Y] \text { and } Y \subset V[Z]\}
$$

One easily checks that the set $\mathcal{B}=\{\widehat{V}: V \in \mathcal{U}\} \subset \mathcal{P}(\mathcal{P}(X) \times \mathcal{P}(X))$ satisfies conditions (B-1)-(B-4). Therefore, there exists a unique uniform structure on $\mathcal{P}(X)$ admitting $\mathcal{B}$ as a base. This uniform structure is called the Hausdorff-Bourbaki uniform structure associated with $\mathcal{U}$. The topology associated with the Hausdorff-Bourbaki uniform structure is called the Hausdorff-Bourbaki topology on $\mathcal{P}(X)$. If $\left(Y_{i}\right)_{i \in I}$ is a net in $\mathcal{P}(X)$ (that is, a family of subsets of $X$ indexed by some directed set $I$ ) and $Z \in \mathcal{P}(X)$, we shall write $Y_{i} \stackrel{H-B}{\rightarrow} Z$ to mean that the net $\left(Y_{i}\right)_{i \in I}$ converges to $Z$ in the Hausdorff-Bourbaki topology.

Proposition 3.1 Let $X$ be a uniform space and let $Y$ and $Z$ be closed subsets of $X$. Suppose that there is a net $\left(T_{i}\right)_{i \in I}$ of subsets of $X$ such that $T_{i} \stackrel{H-B}{\rightarrow} Y$ and $T_{i} \stackrel{H-B}{\rightarrow} Z$. Then one has $Y=Z$.

Proof Let $y \in Y$ and let $\Omega$ be a neighborhood of $y$ in $X$. Then there is a symmetric entourage $V$ of $X$ such that $V[y] \subset \Omega$. Choose an entourage $W$ of $X$ such that $W \circ W \subset V$. Since the net $\left(T_{i}\right)_{i \in I}$ converges to both $Y$ and $Z$, we can find an element $i_{0} \in I$ such that $Y \subset W\left[T_{i_{0}}\right]$ and $T_{i_{0}} \subset W[Z]$. Thus, there exist $t \in T_{i_{0}}$ and $z \in Z$ such that $(y, t) \in W$ and $(t, z) \in W$. This implies $(y, z) \in W \circ W \subset V$ and hence $(z, y) \in V$ since $V$ is symmetric. It follows that $z \in V[y] \subset \Omega$. This shows that $y$ is in the closure of $Z$. Since $Z$ is closed in $X$, we deduce that $Y \subset Z$. By symmetry, we also have $Z \subset Y$. Consequently, $Y=Z$.

Remarks (1) An immediate consequence of Proposition 3.1 is that, if $X$ is a uniform space, then the topology induced by the Hausdorff-Bourbaki topology on the set of closed subsets of $X$ is Hausdorff. 
(2) Suppose that $(X, d)$ is a metric space and let $\mathcal{C}_{b}(X)$ denote the subset of $\mathcal{P}(X)$ consisting of all closed bounded subsets of $X$. For $x \in X$ and $r>0$, denote by $B(x, r)$ the open ball of radius $r$ centered at $x$. The Hausdorff metric is the metric $\delta$ on $\mathcal{C}_{b}(X)$ defined by

$$
\delta(Y, Z)=\inf \left\{r>0: Z \subset \bigcup_{y \in Y} B(y, r) \text { and } Y \subset \bigcup_{z \in Z} B(z, r)\right\}
$$

The uniform structure on $\mathcal{C}_{b}(X)$ associated with the metric $\delta$ is the uniform structure induced by the Hausdorff-Bourbaki structure on $\mathcal{P}(X)$.

Proposition 3.2 Let $X$ be a uniform space and let us equip $\mathcal{P}(X)$ with the HausdorffBourbaki uniform structure and $\mathcal{P}(X) \times \mathcal{P}(X)$ with the corresponding product uniform structure. Then the map $\eta: \mathcal{P}(X) \times \mathcal{P}(X) \rightarrow \mathcal{P}(X)$ defined by $\eta(Y, Z)=Y \cup Z$ is uniformly continuous.

Proof Let $V$ be an entourage of $X$ and consider the entourage $\widehat{V}$ of $\mathcal{P}(X)$ given by (3.1). Suppose that $Y_{1}, Y_{2}, Z_{1}, Z_{2}$ are subsets of $X$ such that $\left(Y_{1}, Y_{2}\right) \in \widehat{V}$ and $\left(Z_{1}, Z_{2}\right) \in \widehat{V}$. Then we have $Y_{1} \subset V\left[Y_{2}\right] \subset V\left[Y_{2} \cup Z_{2}\right]$ and $Z_{1} \subset V\left[Z_{2}\right] \subset V\left[Y_{2} \cup Z_{2}\right]$, and therefore $Y_{1} \cup Z_{1} \subset V\left[Y_{2} \cup Z_{2}\right]$. Similarly, we get $Y_{2} \cup Z_{2} \subset V\left[Y_{1} \cup Z_{1}\right]$. We deduce that $\left(Y_{1} \cup Z_{1}, Y_{2} \cup Z_{2}\right) \in \widehat{V}$. Consequently, we have $(\eta \times \eta)(W) \subset \widehat{V}$, where $W$ is the entourage of $\mathcal{P}(X) \times \mathcal{P}(X)$ defined by

$$
W=\left\{\left(\left(Y_{1}, Z_{1}\right),\left(Y_{2}, Z_{2}\right)\right) \in(\mathcal{P}(X) \times \mathcal{P}(X)) \times(\mathcal{P}(X) \times \mathcal{P}(X)):\left(Y_{i}, Z_{i}\right) \in \widehat{V}(i=1,2)\right\}
$$

This shows that $\eta$ is uniformly continuous.

Corollary 3.3 Let $X$ be a uniform space. Let $\left(Y_{i}\right)_{i \in I}$ and $\left(Z_{i}\right)_{i \in I}$ be nets in $\mathcal{P}(X)$ such that $Y_{i} \subset Z_{i}$ for all $i \in I$. Suppose that there exist closed subsets $A$ and $B$ of $X$ such that $Y_{i} \stackrel{H-B}{\rightarrow} A$ and $Z_{i} \stackrel{H-B}{\rightarrow} B$. Then one has $A \subset B$.

Proof We have $Y_{i} \cup Z_{i} \stackrel{H-B}{\rightarrow} A \cup B$ by continuity of the union map $\eta$. Since $Y_{i} \cup Z_{i}=Z_{i}$ for all $i \in I$, Proposition 3.1 implies that $A \cup B=B$, that is, $A \subset B$.

Proposition 3.4 Let $X$ and $Y$ be uniform spaces and let $f: X \rightarrow Y$ be a uniformly continuous map. Then the map $f_{*}: \mathcal{P}(X) \rightarrow \mathcal{P}(Y)$ which sends each subset $A \subset X$ to its image $f(A) \subset Y$ is uniformly continuous with respect to the Hausdorff-Bourbaki uniform structures on $\mathcal{P}(X)$ and $\mathcal{P}(Y)$.

Proof Let $W$ be an entourage of $Y$ and let

$$
\widehat{W}=\left\{\left(B_{1}, B_{2}\right) \in \mathcal{P}(Y) \times \mathcal{P}(Y): B_{2} \subset W\left[B_{1}\right] \text { and } B_{1} \subset W\left[B_{2}\right]\right\}
$$

be the associated entourage of $\mathcal{P}(Y)$. Since $f$ is uniformly continuous, there is an entourage $V$ of $X$ such that

$$
(f \times f)(V) \subset W .
$$


Suppose that $\left(A_{1}, A_{2}\right) \in \widehat{V}$, that is, $A_{2} \subset V\left[A_{1}\right]$ and $A_{1} \subset V\left[A_{2}\right]$. If $a_{1} \in A_{1}$, then there exists $a_{2} \in A_{2}$ such that $\left(a_{1}, a_{2}\right) \in V$ and hence $\left(f\left(a_{1}\right), f\left(a_{2}\right)\right) \in W$. Therefore, we have $f_{*}\left(A_{1}\right) \subset W\left[f_{*}\left(A_{2}\right)\right]$. Similarly, we get $f_{*}\left(A_{2}\right) \subset W\left[f_{*}\left(A_{1}\right)\right]$. It follows that $\left(f_{*}\left(A_{1}\right), f_{*}\left(A_{2}\right)\right) \in \widehat{W}$. This shows that $\left(f_{*} \times f_{*}\right)(\widehat{V}) \subset \widehat{W}$. Consequently, $f_{*}$ is uniformly continuous.

Corollary 3.5 Let $X$ be a uniform space and let $f: X \rightarrow X$ be a uniformly continuous map. Let $Y$ be a subset of $X$ such that the sets $Y$ and $f(Y)$ are both closed in $X$. Suppose that there is a net $\left(Z_{i}\right)_{i \in I}$ of subsets of $X$ such that $f\left(Z_{i}\right) \subset Z_{i}$ for all $i \in I$ and $Z_{i} \stackrel{H-B}{\rightarrow} Y$. Then one has $f(Y) \subset Y$.

Proof We have $f\left(Z_{i}\right) \subset Z_{i}$ for all $i \in I$. Since $f\left(Z_{i}\right) \stackrel{H-B}{\rightarrow} f(Y)$ by Proposition 3.4, we deduce that $f(Y) \subset Y$ by applying Corollary 3.3.

In particular, we have:

Corollary 3.6 Let $X$ be a uniform space equipped with a uniformly continuous action of a group $\Gamma$. Let $Y$ be a closed subset of $X$. Suppose that there is a net $\left(Z_{i}\right)_{i \in I}$ of $\Gamma$-invariant subsets of $X$ such that $Z_{i} \stackrel{H-B}{\rightarrow} Y$. Then $Y$ is $\Gamma$-invariant.

\section{Gromov's injectivity lemma}

The following result is a uniform version of Lemma 4.H in [14].

Theorem 4.1 (Gromov's injectivity lemma) Let $X$ be a uniform space equipped with a uniformly continuous and expansive action of a group $\Gamma$ and let $f: X \rightarrow X$ be a uniformly continuous and $\Gamma$-equivariant map. Suppose that $Y$ is a subset of $X$ such that the restriction of $f$ to $Y$ is a uniform embedding. Then there exists an entourage $V$ of $X$ satisfying the following property: if $Z$ is a $\Gamma$-invariant subset of $X$ such that $Z \subset V[Y]$, then the restriction of $f$ to $Z$ is injective.

Proof By expansivity of the action of $\Gamma$ on $X$, there exists an entourage $W_{0}$ of $X$ such that

$$
\bigcap_{\gamma \in \Gamma} \gamma^{-1} W_{0}=\Delta_{X} .
$$

It follows from the axioms of a uniform structure that we can find a symmetric entourage $S$ of $X$ such that

$$
S \circ S \circ S \subset W_{0}
$$

Since the restriction of $f$ to $Y$ is a uniform embedding, there exists an entourage $T$ of $X$ such that

$$
\left(f\left(y_{1}\right), f\left(y_{2}\right)\right) \in T \Rightarrow\left(y_{1}, y_{2}\right) \in S
$$


for all $y_{1}, y_{2} \in Y$. Let $U$ be a symmetric entourage of $X$ such that

$$
U \circ U \subset T \text {. }
$$

As $f$ is uniformly continuous, we can find an entourage $E$ of $X$ such that

$$
\left(x_{1}, x_{2}\right) \in E \Rightarrow\left(f\left(x_{1}\right), f\left(x_{2}\right)\right) \in U
$$

for all $x_{1}, x_{2} \in X$.

Let us show that the entourage $V=S \cap E$ has the required property. Suppose that $Z$ is a $\Gamma$-invariant subset of $X$ such that $Z \subset V[Y]$. Let us show that the restriction of $f$ to $Z$ is injective.

Let $z^{\prime}$ and $z^{\prime \prime}$ be points in $Z$ such that $f\left(z^{\prime}\right)=f\left(z^{\prime \prime}\right)$ and let $\gamma \in \Gamma$. Since $f$ is $\Gamma$-equivariant, we have

$$
f\left(\gamma z^{\prime}\right)=f\left(\gamma z^{\prime \prime}\right)
$$

As $\gamma z^{\prime}$ and $\gamma z^{\prime \prime}$ stay in $Z$ and $Z \subset V[Y]$, we can find points $y_{\gamma}^{\prime}$ and $y_{\gamma}^{\prime \prime}$ in $Y$ such that $\left(\gamma z^{\prime}, y_{\gamma}^{\prime}\right) \in V$ and $\left(\gamma z^{\prime \prime}, y_{\gamma}^{\prime \prime}\right) \in V$. Since $V \subset E$, it follows from (4.5) that $\left(f\left(\gamma z^{\prime}\right), f\left(y_{\gamma}^{\prime}\right)\right)$ and $\left(f\left(\gamma z^{\prime \prime}\right), f\left(y_{\gamma}^{\prime \prime}\right)\right)$ are in $U$. As $U$ is symmetric we also have $\left(f\left(y_{\gamma}^{\prime}\right), f\left(\gamma z^{\prime}\right)\right) \in U$ and therefore $\left(f\left(y_{\gamma}^{\prime}\right), f\left(y_{\gamma}^{\prime \prime}\right)\right) \in U \circ U \subset T$ by (4.6) and (4.4). By applying (4.3), we deduce that $\left(y_{\gamma}^{\prime}, y_{\gamma}^{\prime \prime}\right) \in S$. On the other hand, we also have $\left(\gamma z^{\prime}, y_{\gamma}^{\prime}\right) \in S$ and $\left(y_{\gamma}^{\prime \prime}, \gamma z^{\prime \prime}\right) \in S$ since $V \subset S$ and $S$ is symmetric. It follows that

$$
\left(\gamma z^{\prime}, \gamma z^{\prime \prime}\right) \in S \circ S \circ S \subset W_{0}
$$

by (4.2). This gives us

$$
\left(z^{\prime}, z^{\prime \prime}\right) \in \bigcap_{\gamma \in \Gamma} \gamma^{-1} W_{0},
$$

and hence $z^{\prime}=z^{\prime \prime}$ by (4.1). This shows that the restriction of $f$ to $Z$ is injective.

Let $f: X \rightarrow X$ be a map from a set $X$ into itself. If $Y$ is a subset of $X$ such that $f(Y) \subset Y$, we denote by $\left.f\right|_{Y}: Y \rightarrow Y$ the restriction map given by $\left.f\right|_{Y}(y)=f(y)$ for all $y \in Y$.

Corollary 4.2 Let $X$ be a uniform space equipped with a uniformly continuous and expansive action of a group $\Gamma$ and let $f: X \rightarrow X$ be a uniformly continuous and $\Gamma$-equivariant map. Let $Y$ be a subset of $X$ such that $Y$ and $f(Y)$ are both closed in $X$ and the restriction of $f$ to $Y$ is a uniform embedding. Suppose that there exists a net $\left(Z_{i}\right)_{i \in I}$ of $\Gamma$-invariant subsets of $X$ such that $f\left(Z_{i}\right) \subset Z_{i}$ and the restriction maps $\left.f\right|_{Z_{i}}: Z_{i} \rightarrow Z_{i}$ are surjunctive for all $i \in I$ and $Z_{i} \stackrel{H-B}{\rightarrow} Y$. Then $Y$ is $\Gamma$-invariant and one has $f(Y)=Y$. 
Proof The $\Gamma$-invariance of $Y$ directly follows from Corollary 3.6. Let $V$ be an entourage of $X$ as in Theorem 4.1. As $Z_{i} \stackrel{H-B}{\rightarrow} Y$, there is an element $i_{0} \in I$ such that $Z_{i} \subset V[Y]$ for all $i \geq i_{0}$. Using the fact that $\left.f\right|_{Z_{i}}$ is surjunctive, we deduce that $f\left(Z_{i}\right)=Z_{i}$ for all $i \geq i_{0}$. We have $f\left(Z_{i}\right) \stackrel{H-B}{\rightarrow} f(Y)$ by Proposition 3.4. As $Y$ and $f(Y)$ are closed in $X$, we conclude that $f(Y)=Y$ by applying Proposition 3.1.

Proof of Theorem 1.1 First observe that the space $X$ is Hausdorff since it admits an expansive uniformly continuous action. It follows that both $Y$ and $f(Y)$ are closed in $X$ by compactness of $Y$.

The fact that $Y$ is $\Gamma$-invariant and satisfies $f(Y) \subset Y$ is a consequence of Corollaries 3.5 and 3.6.

Suppose now that $\left.f\right|_{Y}$ is injective. As $Y$ is compact, we deduce that the restriction of $f$ is a uniform embedding. We conclude that $\left.f\right|_{Y}$ is surjective by applying Corollary 4.2.

\section{Cellular automata}

Let $G$ be a group and let $A$ be an arbitrary set that we shall call the alphabet. We consider the set $A^{G}$ consisting of all maps $x: G \rightarrow A$. The elements of $A^{G}$ are called configurations over the group $G$ and the alphabet $A$.

Given a subset $S \subset G$, we denote by $\pi_{S}: A^{G} \rightarrow A^{S}$ the projection map.

We equip the set $A^{G}=\prod_{g \in G} A$ with its prodiscrete uniform structure (cf. Sect. 2.3) and the left action of $G$ defined by $g x(h)=x\left(g^{-1} h\right)$ for all $g, h \in G$ and $x \in A^{G}$. This action is called the $G$-shift on $A^{G}$.

Proposition 5.1 The $G$-shift on $A^{G}$ is uniformly continuous and expansive.

Proof Uniform continuity follows from the fact that $G$ acts on $A^{G}$ by permuting coordinates. Expansiveness is due to the fact that the action of $G$ on itself by left multiplication is transitive. Indeed, consider the entourage $W_{0}$ of $A^{G}$ defined by

$$
W_{0}=\left\{(x, y) \in A^{G} \times A^{G}: x\left(1_{G}\right)=y\left(1_{G}\right)\right\} .
$$

Given $g \in G$, we have $(x, y) \in g^{-1} W_{0}$ if and only if $x\left(g^{-1}\right)=y\left(g^{-1}\right)$. Thus $\bigcap_{g \in G} g^{-1} W_{0}$ is equal to the diagonal in $A^{G} \times A^{G}$.

The space $A^{G}$ with the $G$-shift action is called the full shift on $G$ and $A$. A $G$ invariant subset $X \subset A^{G}$ is called a subshift (note that here we do not require $X$ to be closed in $A^{G}$ ).

Definition Let $X, Y \subset A^{G}$ be two subshifts. A cellular automaton from $X$ to $Y$ is a map $\tau: X \rightarrow Y$ satisfying the following property: there exist a finite subset $S \subset G$ and a map $\mu: \pi_{S}(X) \rightarrow A$ such that

$$
\tau(x)(g)=\mu\left(\pi_{S}\left(g^{-1} x\right)\right) \text { for all } x \in X \text { and } g \in G .
$$

Such a set $S$ is then called a memory set and $\mu$ is called a local defining map for $\tau$. 
The following statement is proved in [5] (see also [7]) in the particular case when $X=Y=A^{G}$ :

Proposition 5.2 Let $G$ be a group and let $A$ be a set. Let $X, Y \subset A^{G}$ be two subshifts and let $\tau: X \rightarrow Y$ be a map. Then the following conditions are equivalent:

(a) $\tau$ is a cellular automaton;

(b) $\tau$ is uniformly continuous and $G$-equivariant;

(c) There exists a cellular automaton $\tilde{\tau}: A^{G} \rightarrow A^{G}$ such that $\tilde{\tau}(X) \subset Y$ and $\tau(x)=\widetilde{\tau}(x)$ for all $x \in X$.

Proof Suppose that $\tau$ is a cellular automaton with memory set $S$ and local defining map $\mu: \pi_{S}(X) \rightarrow A$. Let $\tilde{\mu}: A^{S} \rightarrow A$ be a map extending $\mu$. Then the map $\tilde{\tau}: A^{G} \rightarrow A^{G}$ defined by $\tilde{\tau}(x)(g)=\tilde{\mu}\left(\pi_{S}\left(g^{-1} x\right)\right)$ for all $x \in A^{G}$ and $g \in G$ is a cellular automaton over $A^{G}$ such that $\tilde{\tau}(X) \subset Y$ and $\tilde{\tau}(x)=\tau(x)$ for all $x \in X$. This shows that (a) implies (c).

It is shown in [5, Th. 1.1] that every cellular automaton $\tilde{\tau}: A^{G} \rightarrow A^{G}$ is $G$ equivariant and uniformly continuous. Therefore (c) implies (b).

Suppose that $\tau: X \rightarrow Y$ is $G$-equivariant and uniformly continuous. Since the map $f: X \rightarrow A$ defined by $f(x)=\tau(x)\left(1_{G}\right)$ is uniformly continuous, this implies that there is a finite subset $S \subset G$ such that if two elements $x, y \in X$ coincide on $S$ then $f(x)=f(y)$. In other words, there is a map $\mu: \pi_{S}(X) \rightarrow A$ such that $\tau(x)\left(1_{G}\right)=\mu\left(\pi_{S}(x)\right)$ for all $x \in X$. Now the $G$-equivariance of $\tau$ gives us

$$
\tau(x)(g)=g^{-1} \tau(x)\left(1_{G}\right)=\tau\left(g^{-1} x\right)\left(1_{G}\right)=\mu\left(\pi_{S}\left(g^{-1} x\right)\right)
$$

for all $x \in X$ and $g \in G$. This shows that (b) implies (a).

If $X, Y \subset A^{G}$ are two subshifts, we shall denote by $\mathrm{CA}(X, Y ; G, A)$ the set consisting of all cellular automata $\tau: X \rightarrow Y$. If $Y=X$ we shall simply write $\mathrm{CA}(X ; G, A)$ instead of $\mathrm{CA}(X, X ; G, A)$. Note that it immediately follows from the equivalence of conditions (a) and (b) in Proposition 5.2 that $\mathrm{CA}(X ; G, A)$ is a monoid for the composition of maps.

\section{Marked groups}

Let $\Gamma$ be a group. A $\Gamma$-quotient is a pair $(G, \rho)$, where $G$ is a group and $\rho: \Gamma \rightarrow G$ is a group epimorphism. Two $\Gamma$-quotients $\left(G_{1}, \rho_{1}\right)$ and $\left(G_{2}, \rho_{2}\right)$ are said to be equivalent if there exists a group isomorphism $\phi: G_{2} \rightarrow G_{1}$ such that the following diagram is commutative:

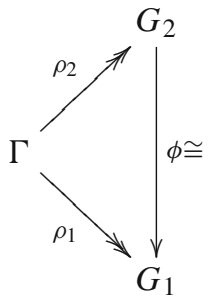


that is, such that $\rho_{1}=\phi \circ \rho_{2}$. An equivalence class of $\Gamma$-quotients is called a $\Gamma$-marked group. Observe that two $\Gamma$-quotients $\left(G_{1}, \rho_{1}\right)$ and $\left(G_{2}, \rho_{2}\right)$ are equivalent if and only if $\operatorname{Ker}\left(\rho_{1}\right)=\operatorname{Ker}\left(\rho_{2}\right)$. Thus the set of $\Gamma$-marked groups may be identified with the set $\mathcal{N}(\Gamma)$ consisting of all normal subgroups of $\Gamma$.

Let us equip the set $\mathcal{P}(\Gamma)=\{0,1\}^{\Gamma}$ with its prodiscrete uniform structure and $\mathcal{N}(\Gamma)$ with the induced uniform structure. A base of entourages of $\mathcal{N}(\Gamma)$ is provided by the sets

$$
V_{F}=\left\{\left(N_{1}, N_{2}\right) \in \mathcal{N}(\Gamma) \times \mathcal{N}(\Gamma): N_{1} \cap F=N_{2} \cap F\right\},
$$

where $F$ runs over all finite subsets of $\Gamma$. Intuitively, two normal subgroups of $\Gamma$ are "close" in $\mathcal{N}(\Gamma)$ when their intersections with a large finite subset of $\Gamma$ coincide.

The space $\mathcal{P}(\Gamma)$ is Hausdorff and totally disconnected since it is a product of discrete spaces. Moreover, $\mathcal{P}(\Gamma)$ is compact by the Tychonoff product theorem. The set $\mathcal{N}(\Gamma)$ is closed in $\mathcal{P}(\Gamma)$ and therefore $\mathcal{N}(\Gamma)$ is a totally disconnected compact Hausdorff space (see [8,9] and the references therein).

Let $A$ be a set. Consider the set $A^{\Gamma}$ equipped with its prodiscrete uniform structure and the $\Gamma$-shift action. For each $N \in \mathcal{N}(\Gamma)$, let

$$
\operatorname{Fix}(N)=\left\{x \in A^{\Gamma}: \gamma x=x \text { for all } \gamma \in N\right\} \subset A^{\Gamma}
$$

denote the set of points in $A^{\Gamma}$ which are fixed by $N$. Observe that Fix $(N)$ is a closed subshift of $A^{\Gamma}$. Since $N$ acts trivially on Fix $(N)$, the $\Gamma$-shift on $A^{\Gamma}$ induces an action of the quotient group $G=\Gamma / N$ on $\operatorname{Fix}(N)$.

Proposition 6.1 Let $N$ be a normal subgroup of a group $\Gamma$ and let $\rho: \Gamma \rightarrow G=\Gamma / N$ denote the canonical epimorphism. Then $y \circ \rho \in \operatorname{Fix}(N)$ for every $y \in A^{G}$. Moreover, the map $\rho^{*}: A^{G} \rightarrow \operatorname{Fix}(N)$ defined by $\rho^{*}(y)=y \circ \rho$ is a $G$-equivariant uniform isomorphism.

Proof An element $x \in A^{\Gamma}$ is in $\operatorname{Fix}(N)$ if and only if $x\left(v^{-1} \gamma\right)=x(\gamma)$ for all $\gamma \in \Gamma$ and $v \in N$, that is, if and only if the configuration $x$ is constant on each coset of $\Gamma$ modulo $N$. This proves the first assertion and the fact that the map $\rho^{*}$ is surjective. The injectivity of $\rho^{*}$ follows from the surjectivity of $\rho$. Let $g \in G$ and choose $\gamma \in \Gamma$ such that $\rho(\gamma)=g$. For all $y \in A^{G}$ and $\alpha \in \Gamma$, we have

$$
\begin{aligned}
g(y \circ \rho)(\alpha) & =\gamma(y \circ \rho)(\alpha)=y \circ \rho\left(\gamma^{-1} \alpha\right)=y\left(\rho\left(\gamma^{-1} \alpha\right)\right)=y\left(g^{-1} \rho(\alpha)\right) \\
& =((g y) \circ \rho)(\alpha) .
\end{aligned}
$$

This shows that $g(y \circ \rho)=(g y) \circ \rho$, that is, $g \rho^{*}(y)=\rho^{*}(g y)$. Thus $\rho^{*}$ is $G$ equivariant.

For each $\gamma \in \Gamma$, let $\pi_{\gamma}: A^{\Gamma} \rightarrow A$ and $\pi_{\gamma}^{\prime}: A^{G} \rightarrow A$ denote the projection maps given by $x \mapsto x(\gamma)$ and $y \mapsto y(\rho(\gamma))$ respectively. The fact that $\pi_{\gamma} \circ \rho^{*}=\pi_{\gamma}^{\prime}$ is uniformly continuous for all $\gamma \in \Gamma$ implies that the map $\rho^{*}$ is uniformly continuous. Similarly, the uniform continuity of $\left(\rho^{*}\right)^{-1}$ follows from the fact that $\pi_{\gamma}^{\prime} \circ\left(\rho^{*}\right)^{-1}=$ $\left.\pi_{\gamma}\right|_{\operatorname{Fix}(N)}$ is uniformly continuous for each $\gamma \in \Gamma$. Consequently, $\rho^{*}$ is a uniform isomorphism. 
By using the characterization of cellular automata provided by the equivalence of conditions (a) and (b) in Proposition 5.2, we immediately deduce the following:

Corollary 6.2 If $\tau: A^{G} \rightarrow A^{G}$ is a cellular automaton over $A^{G}$, then the map $\tau^{*}: \operatorname{Fix}(N) \rightarrow \operatorname{Fix}(N)$ given by $\tau^{*}=\rho^{*} \circ \tau \circ\left(\rho^{*}\right)^{-1}$ is a cellular automaton over the subshift $\operatorname{Fix}(N) \subset A^{\Gamma}$. Moreover, the map $\Theta: \operatorname{CA}\left(A^{G} ; G, A\right) \rightarrow \mathrm{CA}(\operatorname{Fix}(N) ; \Gamma, A)$ defined by $\Theta(\tau)=\tau^{*}$ is a monoid isomorphism.

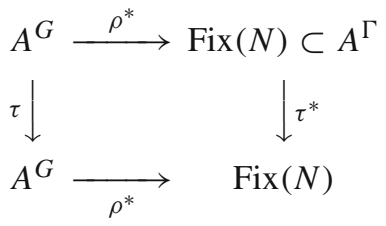

Let us equip $\mathcal{P}\left(A^{\Gamma}\right)$ with the Hausdorff-Bourbaki uniform structure associated with the prodiscrete uniform structure on $A^{\Gamma}$.

Theorem 6.3 Let $\Gamma$ be a group and let $A$ be a set. Then the map $\Psi: \mathcal{N}(\Gamma) \rightarrow \mathcal{P}\left(A^{\Gamma}\right)$ defined by $\Psi(N)=\operatorname{Fix}(N)$ is uniformly continuous. Moreover, if A contains at least two elements then $\Psi$ is a uniform embedding.

Proof Let $N_{0} \in \mathcal{N}(\Gamma)$ and let $W$ be an entourage of $\mathcal{P}\left(A^{\Gamma}\right)$. Let us show that there exists an entourage $V$ of $\mathcal{N}(\Gamma)$ such that

$$
\Psi\left(V\left[N_{0}\right]\right) \subset W\left[\Psi\left(N_{0}\right)\right]
$$

This will prove that $\Psi$ is continuous. By definition of the Hausdorff-Bourbaki uniform structure on $\mathcal{P}\left(A^{\Gamma}\right)$, there is an entourage $T$ of $A^{\Gamma}$ such that

$$
\widehat{T}=\left\{(X, Y) \in \mathcal{P}\left(A^{\Gamma}\right) \times \mathcal{P}\left(A^{\Gamma}\right): Y \subset T[X] \text { and } X \subset T[Y]\right\} \subset W
$$

Since $A^{\Gamma}$ is endowed with its prodiscrete uniform structure, there is a finite subset $F \subset \Gamma$ such that

$$
U=\left\{(x, y) \in A^{\Gamma} \times A^{\Gamma}: \pi_{F}(x)=\pi_{F}(y)\right\} \subset T,
$$

where $\pi_{F}: A^{\Gamma} \rightarrow A^{F}$ is the projection map. Consider now the finite subset $E \subset \Gamma$ defined by

$$
E=F \cdot F^{-1}=\left\{\gamma \eta^{-1}: \gamma, \eta \in F\right\}
$$

and the entourage $V$ of $\mathcal{N}(\Gamma)$ given by

$$
V=\left\{\left(N_{1}, N_{2}\right) \in \mathcal{N}(\Gamma) \times \mathcal{N}(\Gamma): N_{1} \cap E=N_{2} \cap E\right\} .
$$


We claim that $V$ satisfies (6.1). To prove our claim, suppose that $N \in V\left[N_{0}\right]$. Let $x \in \operatorname{Fix}(N)$. The fact that $N \cap E=N_{0} \cap E$ implies that if $\gamma$ and $\eta$ are elements of $F$ with $\gamma=v \eta$ for some $v \in N$, then $v \in N_{0}$ and therefore $x(\gamma)=x(\eta)$. Denoting by $\rho_{0}: \Gamma \rightarrow \Gamma / N_{0}$ the canonical epimorphism, we deduce that we may find an element $x_{0} \in A^{\Gamma / N_{0}}$ such that $x(\gamma)=x_{0} \circ \rho_{0}(\gamma)$ for all $\gamma \in F$. We have $x_{0} \circ \rho_{0} \in \operatorname{Fix}\left(N_{0}\right)$ and $\left(x, x_{0} \circ \rho_{0}\right) \in U$. Since $U \subset T$ by (6.3), this shows that $\operatorname{Fix}(N) \subset T\left[\operatorname{Fix}\left(N_{0}\right)\right]$. Therefore $\Psi$ is continuous and hence uniformly continuous by compactness of $\mathcal{N}(\Gamma)$.

Suppose now that $A$ has at least two elements. Let us show that $\Psi$ is injective. Let $N_{1}, N_{2} \in \mathcal{N}(\Gamma)$. Fix two elements $a, b \in A$ with $a \neq b$ and consider the map $x: \Gamma \rightarrow A$ defined by $x(\gamma)=a$ if $\gamma \in N_{1}$ and $x(\gamma)=b$ otherwise. We clearly have $x \in \operatorname{Fix}\left(N_{1}\right)$. Suppose that $\Psi\left(N_{1}\right)=\Psi\left(N_{2}\right)$, that is, $\operatorname{Fix}\left(N_{1}\right)=\operatorname{Fix}\left(N_{2}\right)$. Then for all $v \in N_{2}$, we have $v^{-1} x=x$ since $x \in \operatorname{Fix}\left(N_{1}\right)=\operatorname{Fix}\left(N_{2}\right)$, and hence $x(v)=v^{-1} x\left(1_{\Gamma}\right)=x\left(1_{\Gamma}\right)=a$. This implies $N_{2} \subset N_{1}$. By symmetry, we also have $N_{1} \subset N_{2}$. Therefore $N_{1}=N_{2}$. This shows that $\Psi$ is injective. As $\mathcal{N}(\Gamma)$ is compact and $\mathcal{P}\left(A^{\Gamma}\right)$ is Hausdorff, we conclude that $\Psi$ is a uniform embedding.

Proof of Corollary 1.3 Let $N \in \mathcal{N}(\Gamma)$ and let $\left(N_{i}\right)_{i \in I}$ be a net in $\mathcal{N}(\Gamma)$ converging to $N$. Suppose that the groups $G_{i}=\Gamma / N_{i}$ are surjunctive for all $i \in I$ and let us show that the group $G=\Gamma / N$ is surjunctive as well.

Let $A$ be a finite set and let $\tau: A^{G} \rightarrow A^{G}$ be a cellular automaton over $A^{G}$. Let $\rho: \Gamma \rightarrow G$ denote the canonical epimorphism and let $\rho^{*}: A^{G} \rightarrow \operatorname{Fix}(N)$ be the map defined by $\rho^{*}=y \circ \rho$ for all $y \in A^{G}$ (cf. Proposition 6.1). By Corollary 6.2, the map $\tau^{*}: \operatorname{Fix}(N) \rightarrow \operatorname{Fix}(N)$ given by $\tau^{*}=\rho^{*} \circ \tau \circ\left(\rho^{*}\right)^{-1}$ is a cellular automaton over the subshift $\operatorname{Fix}(N) \subset A^{\Gamma}$. By applying Proposition 5.2, we deduce the existence of a cellular automaton $\tilde{\tau}: A^{\Gamma} \rightarrow A^{\Gamma}$ such that $\tilde{\tau}(\operatorname{Fix}(N)) \subset \operatorname{Fix}(N)$ and $\left.\tilde{\tau}\right|_{\operatorname{Fix}(N)}=\tau^{*}$.

We claim that the hypotheses of Theorem 1.1 are satisfied by taking $X=A^{\Gamma}$, $f=\tilde{\tau}, Y=\operatorname{Fix}(N)$, and $Z_{i}=\operatorname{Fix}\left(N_{i}\right)$. First observe that the space $X$ is compact by the Tychonoff product theorem since $A$ is finite. As $Y$ is closed in $X$, it follows that $Y$ is compact. Moreover, the action of $\Gamma$ on $X$ is uniformly continuous and expansive by Proposition 5.1. On the other hand, the cellular automaton $f: X \rightarrow X$ is uniformly continuous and $\Gamma$-equivariant by Proposition 5.2. We have $f\left(Z_{i}\right) \subset Z_{i}$ since the cellular automaton $f$ is $\Gamma$-equivariant. Moreover, $Z_{i}=\operatorname{Fix}\left(N_{i}\right)$ is $\Gamma$-invariant by normality of the subgroup $N_{i} \subset \Gamma$. Also, by Theorem 6.3, $Z_{i} \stackrel{H-B}{\rightarrow} Y$ since $N_{i}$ converges to $N$. For $i \in I$, let $\rho_{i}: \Gamma \rightarrow G_{i}=\Gamma / N_{i}$ denote the canonical epimorphism and let $\rho_{i}^{*}: A^{G_{i}} \rightarrow \operatorname{Fix}\left(N_{i}\right)$ be the uniform isomorphism given by Proposition 6.1. It follows from Corollary 6.2 that the map $\tau_{i}=\left.\left(\rho_{i}^{*}\right)^{-1} \circ \tilde{\tau}\right|_{Z_{i}} \circ \rho_{i}^{*}: A^{G_{i}} \rightarrow A^{G_{i}}$ is a cellular automaton and hence is surjunctive since $G_{i}$ is a surjunctive group. This implies that $\left.f\right|_{Z_{i}}=\left.\tilde{\tau}\right|_{\operatorname{Fix}\left(N_{i}\right)}$ is surjunctive as well. By applying Theorem 1.1, we deduce that $\left.f\right|_{Y}=\tau^{*}$ and hence $\tau=\left(\rho^{*}\right)^{-1} \circ \tau^{*} \circ \rho^{*}$ is surjunctive. Consequently, the group $G$ is surjunctive.

\section{Linear cellular automata}

Let $G$ be a group and let $V$ be a vector space over a field $\mathbb{K}$. The set $V^{G}$ has a natural vector space structure and the $G$-shift action on $V^{G}$ is linear (here and in the sequel "linear" stands for " $\mathbb{K}$-linear"). 
A linear subshift of $V^{G}$ is a $G$-invariant vector subspace of $V^{G}$.

Let $X, Y \subset V^{G}$ be two linear subshifts. A linear cellular automaton from $X$ to $Y$ is a cellular automaton $\tau: X \rightarrow Y$ which is linear. Note that if $\tau: X \rightarrow Y$ is a cellular automaton with memory set $S \subset G$ and local defining map $\mu: \pi_{S}(X) \rightarrow V$, then $\tau$ is linear if and only if $\mu$ is linear.

Proposition 7.1 Let $G$ be a group and let $V$ be a vector space over a field $\mathbb{K}$. Let $X, Y \subset V^{G}$ be two linear subshifts and let $\tau: X \rightarrow Y$ be a map. Then the following conditions are equivalent:

(a) $\tau$ is a linear cellular automaton;

(b) $\tau$ is linear, uniformly continuous (with respect to the uniform structures on $X$ and $Y$ induced by the prodiscrete uniform structure on $V^{G}$ ), and $G$-equivariant;

(c) $\tau$ is linear, continuous (with respect to the topologies on $X$ and $Y$ induced by the prodiscrete topology on $V^{G}$ ), and $G$-equivariant;

(d) $\tau$ is linear, continuous (with respect to the topologies on $X$ and $Y$ induced by the prodiscrete topology on $V^{G}$ ) at the constant configuration $x=0$, and $G$ equivariant;

(e) there exists a linear cellular automaton $\tilde{\tau}: V^{G} \rightarrow V^{G}$ such that $\tilde{\tau}(X) \subset Y$ and $\tau(x)=\tilde{\tau}(x)$ for all $x \in X$.

Proof The equivalences (a) $\Leftrightarrow$ (b) $\Leftrightarrow$ (e) immediately follow from Proposition 5.2 after observing that if $S \subset G$ then any linear map $\mu: \pi_{S}(X) \rightarrow V$ may be extended to a linear map $\widetilde{\mu}: V^{S} \rightarrow V$. Since the topology associated with the prodiscrete uniform structure is the prodiscrete topology (cf. Example (1) in Sect. 2.1) and every uniformly continuous map is continuous (cf. Sect. 2.2), we also have (b) $\Rightarrow$ (c). The implication (c) $\Rightarrow$ (d) is trivial. Let us show that (d) $\Rightarrow$ (a). Suppose that $\tau$ is linear, continuous at 0 , and $G$-equivariant. Then, the map $X \rightarrow V$ defined by $x \mapsto \tau(x)\left(1_{G}\right)$ is continuous at 0 since the projection maps $X \rightarrow V$ defined by $x \mapsto x(g)$ are continuous for all $g \in G$ and the composition of continuous maps is continuous. We deduce that there exists a finite subset $S \subset G$ such that if $x \in X$ satisfies $x(s)=0$ for all $s \in S$, then $\tau(x)\left(1_{G}\right)=0$. By linearity, we have that if two configurations $x$ and $y$ coincide on $S$ then $\tau(x)\left(1_{G}\right)=\tau(y)\left(1_{G}\right)$. Thus there exists a linear map $\mu: \pi_{S}(X) \rightarrow V$ such that $\tau(x)\left(1_{G}\right)=\mu\left(\pi_{S}(x)\right)$ for all $x \in X$. As $\tau$ is $G$-equivariant, we deduce that $\tau(x)(g)=\tau\left(g^{-1} x\right)\left(1_{G}\right)=\mu\left(\pi_{S}\left(g^{-1} x\right)\right)$ for all $x \in X$ and $g \in G$. This shows that $\tau$ is the (linear) cellular automaton with memory set $S$ and local defining map $\mu$. Thus (d) implies (a).

We shall denote by $\operatorname{LCA}(X, Y ; G, V)$ the set consisting of all linear cellular automata $\tau: X \rightarrow Y$. If $X=Y$ we shall simply write $\operatorname{LCA}(X ; G, V)$ instead of $\operatorname{LCA}(X, X ; G, V)$. Note that $\operatorname{LCA}(X ; G, V)$ has a natural structure of $\mathbb{K}$-algebra, where the multiplicative law is given by composition.

If $N$ is a normal subgroup of a group $\Gamma$ and $V$ is a vector space over a filed $\mathbb{K}$, then the set $\operatorname{Fix}(N)$ of points of $V^{\Gamma}$ which are fixed by $N$ is a closed linear subshift of $V^{\Gamma}$. From Proposition 6.1, we immediately deduce the following:

Proposition 7.2 Let $N$ be a normal subgroup of a group $\Gamma$ and let $\rho: \Gamma \rightarrow G=\Gamma / N$ denote the canonical epimorphism. Let $V$ be a vector space over a field $\mathbb{K}$. Then 
$y \circ \rho \in \operatorname{Fix}(N)$ for every $y \in V^{G}$. Moreover, the map $\rho^{*}: V^{G} \rightarrow \operatorname{Fix}(N)$ defined by $\rho^{*}(y)=y \circ \rho$ is a linear G-equivariant uniform isomorphism.

Corollary 7.3 If $\tau: V^{G} \rightarrow V^{G}$ is a linear cellular automaton over $V^{G}$, then the map $\tau^{*}: \operatorname{Fix}(N) \rightarrow \operatorname{Fix}(N)$ given by $\tau^{*}=\rho^{*} \circ \tau \circ\left(\rho^{*}\right)^{-1}$ is a linear cellular automaton over the linear subshift $\operatorname{Fix}(N) \subset V^{\Gamma}$. Moreover, the map $\Theta: \operatorname{LCA}\left(V^{G} ; G, V\right) \rightarrow$ $\operatorname{LCA}(\operatorname{Fix}(N) ; \Gamma, V)$ defined by $\Theta(\tau)=\tau^{*}$ is an isomorphism of $\mathbb{K}$-algebras.

In the proof of Theorem 1.4 we shall use the following results.

Lemma 7.4 (Closure lemma) Let $G$ be a group and let $V$ be a finite dimensional vector space over a field $\mathbb{K}$. Let $\tau: V^{G} \rightarrow V^{G}$ be a linear cellular automaton. Then $\tau\left(V^{G}\right)$ is closed in $V^{G}$ for the prodiscrete topology.

Proof When $G$ is countable this is shown in [3, Lemma 3.1]. The general case is treated in [6, Corollary 1.6].

Lemma 7.5 Let $G$ be a group and let $V$ be a finite dimensional vector space over a field $\mathbb{K}$. Let $\tau: V^{G} \rightarrow V^{G}$ be an injective linear cellular automaton and let $Y=\tau\left(V^{G}\right)$ denote the image of $\tau$. Then the inverse map $\tau^{-1}: Y \rightarrow V^{G}$ is a linear cellular automaton and $\tau$ is a uniform embedding.

Proof When $G$ is countable this is shown in [4, Theorem 3.1].

We now treat the general case. Let $S \subset G$ be a memory set for $\tau$ and denote by $\mu: V^{S} \rightarrow V$ the corresponding local defining map. Let $H \subset G$ be the subgroup generated by $S$. Note that $H$ is countable. Let $G / H$ denote the set of all left cosets of $H$ in $G$. For $c \in G / H$ denote by

$$
\pi_{c}: V^{G} \rightarrow V^{c}=\prod_{g \in c} V
$$

the projection map. We have $V^{G}=\prod_{c \in G / H} V^{c}$ and, for every $x \in V^{G}$ we write $x=\left(x_{c}\right)_{c \in G / H}$, where $x_{c}=\pi_{c}(x) \in V^{c}$.

For $c \in G / H, g \in c$ and $z \in V^{c}$ we denote by $g^{-1} z \in V^{H}$ the configuration defined by

$$
\left(g^{-1} z\right)(h)=z(g h)
$$

for all $h \in H$. Then, if $x=\left(x_{c}\right)_{c \in G / H} \in V^{G}$ and $c \in G / H$, we have

$$
\left(g^{-1} x_{c}\right)(h)=x_{c}(g h)=x(g h)=\left(g^{-1} x\right)(h)=\left(g^{-1} x\right)_{H}(h)
$$

for all $h \in H$, that is,

$$
g^{-1} x_{c}=\left(g^{-1} x\right)_{H}
$$

For $c \in G / H$, define the map $\tau_{c}: V^{c} \rightarrow V^{c}$ by setting

$$
\tau_{c}(z)(g)=\mu\left(\pi_{S}^{H}\left(g^{-1} z\right)\right)
$$


for all $z \in V^{c}$ and $g \in c$, where $\pi_{S}^{H}: V^{H} \rightarrow V^{S}$ is the projection map. Observe that $\tau_{H}: V^{H} \rightarrow V^{H}$ is a linear cellular automaton with memory set $S$ over the group $H$. We then have

$$
\tau=\prod_{c \in G / H} \tau_{c}
$$

in the sense that $\pi_{c} \circ \tau=\tau_{c} \circ \pi_{c}$ for all $c \in G / H$.

From (7.2) we immediately deduce that $Y=\prod_{c \in G / H} Y_{c}$, where $Y_{c}=\pi_{c}(Y)=$ $\tau_{c}\left(V^{c}\right)$, and that $\tau_{c}$ is injective for all $c \in G / H$.

Consider the inverse map $\left(\tau_{H}\right)^{-1}: Y_{H} \rightarrow V^{H}$. By countability of $H$, we have by [4, Theorem 3.1] that $\left(\tau_{H}\right)^{-1}$ is a linear cellular automaton. Let $T \subset H$ be a memory set for $\left(\tau_{H}\right)^{-1}$ and let $v: \pi_{T}^{H}\left(Y_{H}\right)=\pi_{T}(Y) \rightarrow V$ be the corresponding local defining map, where $\pi_{T}^{H}: V^{H} \rightarrow V^{T}$ is the projection map. Consider the linear cellular automaton $\sigma: Y \rightarrow V^{G}$ defined by setting

$$
\sigma(y)(g)=v\left(\pi_{T}\left(g^{-1} y\right)\right)
$$

for all $y \in Y$ and $g \in G$. Note that if $\sigma_{c}: Y_{c} \rightarrow V^{c}$ is the map defined by setting $\sigma_{c}(z)(g)=v\left(\pi_{T}^{H}\left(g^{-1} z\right)\right)$ for all $z \in Y_{c}$ and $g \in c$ then $\sigma=\prod_{c \in G / H} \sigma_{c}$. In particular, we have

$$
\sigma_{H}=\left(\tau_{H}\right)^{-1}
$$

For all $c \in G / H$ one has $\sigma_{c}=\left(\tau_{c}\right)^{-1}$. Indeed, given $c \in G / H, z \in V^{c}$ and $g \in c$, we have

$$
\begin{aligned}
\left(\sigma_{c} \circ \tau_{c}\right)(z)(g) & =\sigma_{c}\left(\tau_{c}(z)\right)(g) \\
& =g^{-1} \sigma_{c}\left(\tau_{c}(z)\right)\left(1_{G}\right) \\
(\text { by }(7.1)) & =\sigma_{H}\left(g^{-1} \tau_{c}(z)\right)\left(1_{G}\right) \\
(\operatorname{again} b y(7.1)) & =\sigma_{H}\left(\tau_{H}\left(g^{-1} z\right)\right)\left(1_{G}\right) \\
(\text { by }(7.3)) & =\left(g^{-1} z\right)\left(1_{G}\right) \\
& =z(g) .
\end{aligned}
$$

This shows that $\sigma_{c} \circ \tau_{c}$ equals the identity map $\operatorname{Id}_{V^{c}}: V^{c} \rightarrow V^{c}$.

We have proved that $\tau^{-1}=\sigma: Y \rightarrow V^{G}$ is a linear cellular automaton. In particular, $\tau^{-1}$ is uniformly continuous by Proposition 5.2 and therefore $\tau$ is a uniform embedding.

Proof of Theorem 1.4 Let $N \in \mathcal{N}(\Gamma)$ and let $\left(N_{i}\right)_{i \in I}$ be a net in $\mathcal{N}(\Gamma)$ converging to $N$. Suppose that the groups $G_{i}=\Gamma / N_{i}$ are $\mathrm{L}_{\mathbb{K}}$-surjunctive for all $i \in I$ and let us show that the group $G=\Gamma / N$ is also $\mathrm{L}_{\mathbb{K}}$-surjunctive.

Let $V$ be a finite-dimensional vector space over the field $\mathbb{K}$ and let $\tau: V^{G} \rightarrow V^{G}$ be an injective linear cellular automaton over $V^{G}$. Let $\rho^{*}: V^{G} \rightarrow \operatorname{Fix}(N)$ be the linear uniform isomorphism (cf. Proposition 7.2) given by defined by $\rho^{*}=y \circ \rho$ for all 
$y \in V^{G}$, where $\rho: \Gamma \rightarrow G$ is the canonical epimorphism. By Corollary 7.3, the map $\tau^{*}: \operatorname{Fix}(N) \rightarrow \operatorname{Fix}(N)$ given by $\tau^{*}=\rho^{*} \circ \tau \circ\left(\rho^{*}\right)^{-1}$ is a linear cellular automaton over the linear subshift $\operatorname{Fix}(N) \subset V^{\Gamma}$. By applying Proposition 7.1, we deduce the existence of a linear cellular automaton $\widetilde{\tau}: V^{\Gamma} \rightarrow V^{\Gamma}$ such that $\widetilde{\tau}(\operatorname{Fix}(N)) \subset \operatorname{Fix}(N)$ and $\left.\widetilde{\tau}\right|_{\operatorname{Fix}(N)}=\tau^{*}$.

We claim that the hypotheses of Corollary 4.2 are satisfied by taking $X=V^{\Gamma}$, $f=\tilde{\tau}, Y=\operatorname{Fix}(N)$, and $Z_{i}=\operatorname{Fix}\left(N_{i}\right)$. The action of $\Gamma$ on $X$ is uniformly continuous and expansive by Proposition 5.1. Also, the linear cellular automaton $f: X \rightarrow X$ is uniformly continuous and $\Gamma$-equivariant by $\operatorname{Proposition} 7.1$. The space $Y=\operatorname{Fix}(N)$ is closed in $X$. On the other hand, $\tau\left(V^{G}\right)$ is closed in $V^{G}$ by virtue of Lemma 7.4, so that $f(Y)=\widetilde{\tau}(\operatorname{Fix}(N))=\tau^{*}(\operatorname{Fix}(N))$ is closed in $Y=\operatorname{Fix}(N)$ and therefore in $X$. As $\tau$ is a injective linear cellular automaton, it follows from Lemma 7.5 that $\tau$ is a uniform embedding of $V^{G}$ into itself. Since $\rho^{*}: V^{G} \rightarrow \operatorname{Fix}(N)$ is a linear uniform isomorphism by Proposition 7.2, we deduce that $\left.f\right|_{Y}=\left.\widetilde{\tau}\right|_{\operatorname{Fix}(N)}=\tau^{*}=\rho^{*} \circ \tau \circ\left(\rho^{*}\right)^{-1}$ is a uniform embedding. We have $f\left(Z_{i}\right) \subset Z_{i}$ since the linear cellular automaton $f$ is $\Gamma$-equivariant. Moreover, $Z_{i}$ is $\Gamma$-invariant by normality of the subgroup $N_{i} \subset \Gamma$. By Theorem 6.3, we have $Z_{i} \stackrel{H-B}{\rightarrow} Y$ since $N_{i}$ converges to $N$. For $i \in I$, let $\rho_{i}: \Gamma \rightarrow G_{i}=\Gamma / N_{i}$ denote the canonical epimorphism and let $\rho_{i}^{*}: V^{G_{i}} \rightarrow \operatorname{Fix}\left(N_{i}\right)$ be the uniform isomorphism given by Proposition 7.2. It follows from Corollary 7.3 that the map $\tau_{i}=\left.\left(\rho_{i}^{*}\right)^{-1} \circ \widetilde{\tau}\right|_{Z_{i}} \circ \rho_{i}^{*}: V^{G_{i}} \rightarrow V^{G_{i}}$ is a linear cellular automaton and hence is surjunctive since $G_{i}$ is an $\mathrm{L}_{\mathbb{K}}$-surjunctive group. This implies that $\left.f\right|_{Z_{i}}=\widetilde{\tau}_{\mathrm{Fix}(N)}$ is surjunctive as well. By applying Corollary 4.2, we deduce that $f(Y)=Y$. Therefore $\tau$ is surjective. This shows that the group $G$ is $\mathrm{L}_{\mathbb{K}}$-surjunctive.

Acknowledgments We express our gratitude to the referee for her/his suggestions and remarks.

Open Access This article is distributed under the terms of the Creative Commons Attribution License which permits any use, distribution and reproduction in any medium, provided the original author(s) and source are credited.

\section{References}

1. Ax, J.: Injective endomorphisms of varieties and schemes. Pacific J. Math. 31, 1-7 (1969)

2. Bourbaki, N.: Topologie générale, Chapitres 1 à 4. Hermann, Paris (1971)

3. Ceccherini-Silberstein, T., Coornaert, M.: The Garden of Eden theorem for linear cellular automata. Ergod. Theory Dyn. Syst. 26, 53-68 (2006)

4. Ceccherini-Silberstein, T., Coornaert, M.: Injective linear cellular automata and sofic groups. Israel J. Math. 161, 1-15 (2007)

5. Ceccherini-Silberstein, T., Coornaert, M.: A generalization of the Curtis-Hedlund theorem. Theor. Comput. Sci. 400, 225-229 (2008)

6. Ceccherini-Silberstein, T., Coornaert, M.: Induction and restriction of cellular automata. Ergod. Theory Dyn. Syst. 29, 371-380 (2009)

7. Ceccherini-Silberstein, T., Coornaert, M.: Cellular Automata and Groups, Springer Monographs in Mathematics. Springer, Berlin (2010)

8. Champetier, C.: L'espace des groupes de type fini. Topology 39, 657-680 (2000)

9. de Cornulier, Y., Guyot, L., Pitsch, W.: On the isolated points in the space of groups. J. Algebra 307, 254-277 (2007)

10. Elek, G., Szabó, A.: Sofic groups and direct finiteness. J. Algebra 280, 426-434 (2004) 
11. Elek, G., Szabó, A.: On sofic groups. J. Group Theory 9, 161-171 (2006)

12. Glebsky, L.Yu., Gordon, E.I.: On surjunctivity of the transition functions of cellular automata on groups. Taiwan. J. Math. 9, 511-520 (2005)

13. Gottschalk, W.: Some general dynamical systems. In: Recent Advances in Topological Dynamics. Lecture Notes in Mathematics, vol. 318, pp. 120-125. Springer, Berlin (1973)

14. Gromov, M.: Endomorphisms of symbolic algebraic varieties. J. Eur. Math. Soc. 1, 109-197 (1999)

15. James, I.M.: Introduction to Uniform Spaces. London Mathematical Society Lecture Note Series, vol. 144. Cambridge University Press, Cambridge (1990)

16. Kaplansky, I.: Fields and Rings. Chicago Lectures in Mathematics. University of Chicago Press, Chicago (1969)

17. Kelley, J.L.: General Topology, Graduate Texts in Mathematics, vol. 27. Springer, New York (1975)

18. Weil, A.: Sur les espaces à structure uniforme et sur la topologie générale. Hermann, Paris (1938)

19. Weiss, B.: Sofic groups and dynamical systems, (Ergodic theory and harmonic analysis, Mumbai, 1999) Sankhya Ser. A. 62, 350-359 (2000) 